EPJ Web of Conferences 59, 02010 (2013)

DOI: $10.1051 /$ epjconf/20135902010

(C) Owned by the authors, published by EDP Sciences, 2013

\title{
Reproducibility of hohlraum-driven implosion symmetry on the National Ignition Facility
}

\author{
G.A. Kyrala1 ${ }^{1}$, D.K. Bradley², D.A. Callahan², S.N. Dixit², M.J. Edwards², \\ S.M. Glenn², S.H. Glenzer², N. Izumi², O.S. Jones², J.L. Kline' ${ }^{1}$, O.L. Landen², \\ T. $\mathrm{Ma}^{2}$, J.L. Milovich², N.B. Meezan², B.K. Spears², R.P.J. Town², S. Weber², \\ R. Benedetti ${ }^{2}$, T. Döppner², J. Ralph ${ }^{2}$ and J. Kilkenny ${ }^{3}$
}

${ }^{1}$ Los Alamos National Laboratory, Los Alamos, NM 87545, USA

${ }^{2}$ Lawrence Livermore National Laboratory, Livermore, CA 94551, USA

${ }^{3}$ General Atomics, San Diego, CA 92121, USA

\begin{abstract}
Indirectly driven Symcap capsules are used at the NIF to obtain information about ignition capsule implosion performance, in particular shape. Symcaps replace the cryogenic fuel layer with an equivalent ablator mass and can be similarly diagnosed. Symcaps are good symmetry surrogates to an ignition capsule after the peak of the drive, radiation-hydrodynamics simulations predict that doping of the symcaps vary the behavior of the implosion. We compare the equatorial shapes of a symcap doped with $\mathrm{Si}$ or Ge, as well as examine the reproducibility of the shape measurement using two symcaps with the same hohlraum and laser conditions.
\end{abstract}

\section{INTRODUCTION}

To successfully achieve inertial confinement fusion (ICF) in the laboratory with minimal energy, we require maximum compression to sizes a few times an alpha particle range in a central spot heated to an ion temperature close to $10 \mathrm{keV}$. These conditions necessitate the use of spherical implosions that have minimal surface losses for a given volume size, and require exquisite spherical symmetry to minimize the energy loss at the surface and to maximize the compression of an initial volume. These compressions must be achieved over a very short time comparable to few times the sound speed propagation across the hot core. To achieve the smallest core with the maximum density, for a given initial mass, requires a large convergence ratio to use the least mass for a given alpha particle range. Thus the core must be as spherical as possible, reducing yield-loss due to surface perturbations [1].

Considerable amount of studies have been performed to specify tolerable low-order mode perturbation amplitudes that reduce the core-size. But measuring such amplitudes in ignition capsules is practically difficult due to the expected large neutron signals that occur while tuning. Alternative capsules, symcaps [2], are ignition capsules where the cryogenic fuel layer in the ignition capsule is replaced by an equivalent mass of ablator material, have the same hydrodynamic behavior as ignition capsules, and have been used to measure the low-order symmetry of the implosion at low nuclear yield. Symcaps, for these experiments, are filled with $30 \% \mathrm{D}_{2}$ and $70 \%{ }^{3} \mathrm{He}$ to take advantage of the neutron and proton yields as diagnostics, and to give measureable x-ray signals to diagnose the implosion. Symcaps, however, while they have similar mode-amplitude variation with perturbations as an ignition capsule, do not converge as much as ignition capsules. Symcaps doped with Ge were found to implode with a smaller velocity than calculated. Si was substituted for the Ge in some of the capsules to find if

This is an Open Access article distributed under the terms of the Creative Commons Attribution License 2.0, which permits unrestricted use, distribution, and reproduction in any medium, provided the original work is properly cited. 


\section{EPJ Web of Conferences}

they converge faster, hence it was necessary to compare the implosion shapes after that change to assess if the tuning that was done with Ge still applies. In what follows we will examine the repeatability of the shape measurements using the same symcap with Ge doping, and later examine the effect of replacing the Ge-dopant with Si in the ablator.

\section{SETUP}

Ignition is sensitive to core size and shape, hence to the ablator-shape at maximum compression. However, the shell is a weak x-ray emitter of high energy x-rays, since little internal work is done on it, other than due to shocks and weak fluorescence of the Ge dopants pumped by either core emission or by hot electrons and their Bremsstrahlung. However, the core heated by PdV work of the imploding shell, is a strong X-ray emitter. Studies had shown the shape of the core, for X-rays above 5-6 keV, correlates well with the shape of the inner surface of the ablator $[3,4]$. We use gated $x$-ray detectors to image the emission. This works well when the nuclear neutron yield is lower than $3 \times 10^{13}$, for CCD-based instruments with computer control, and up to $2 \times 10^{15}$ when using film based, non-computer controlled, gated instruments placed within 1.2-meter from the target [5]. At higher yield new instruments are being developed and will be placed outside the NIF target chamber.

The experimental layout is well described in earlier publications [6-8]. Briefly, $2 \mathrm{~mm}$ diameter capsules with different fills are placed at the center of a $10.1 \mathrm{~mm}$ long, $5.44 \mathrm{~mm}$ or $5.75 \mathrm{~mm}$ diameter cylindrical hohlraum [9]. They are irradiated by laser light at $351 \mathrm{~nm}$ from 192 laser beams, smoothed using polarization smoothing, $45 \mathrm{GHz}$ smoothing by Spectral Dispersion and Continuum Phase Plates [10]. These beams are configured in four cones: two inner cones at 23.5 and 30 degrees with respect to the hohlraum axis, and two outer cones at 44.5 and 50 degrees, enter the hohlraum through $3.1 \mathrm{~mm}$ diameter laser entrance hole [11]. The total laser energy and power are measured with $\pm 2 \%$ and $\pm 3 \%$ accuracy, respectively. Tuning the symmetry is achieved, in these experiments, by varying the internal cone fraction $(\mathrm{CF})$, the ratio of energy in the inner cone to the total energy, inside the hohlraum. This is controlled through variation of the laser wavelength difference between the inner and outer beam cones [7] keeping the external energy and external cone fraction fixed.

\section{RESULTS}

\subsection{Repeatability}

The large number of shots required for tuning an ignition capsule leaves few chances to check repeatability and accuracy of the measurement. However, two Ge-doped symcap shots N110214 and N110612 were taken 4 months apart, with the same target dimensions, and total laser energy, 1.3 MJ. They had a slight difference in the incident foot external CF from $13.5 \%$ to $12.9 \%$ respectively, and with peak external $\mathrm{CF}$ of 0.336 and 0.342 respectively. The equatorial data showed that the implosion time, defined as the time of peak x-ray emission, differ by $80 \mathrm{ps}$, of the order of the $70 \mathrm{ps}$ uncertainty using the GXD time-gated diagnostics. The shape of the implosions is characterized using a Legendre expansion of the shape of the $17 \%$ contour of the emission [4]. The lowest order mode amplitude $\mathrm{P}_{0}$, characterizes the "size" of the implosion. The images for these Ge doped symcap capsules showed some bright spots that could distort the analysis. The bright spots were identified as Ge emission from injected Ge from the ablator [12]. A roller ball is used to locally remove the effect of the Ge bright spots and filter the image, before analysis. Such emission from Ge was reduced significantly in all later NIF shots, which we do not cover here. The data was processed using different techniques to remove the effect of bright emission spots from mixed Ge on core emission. Figure 1 shows a comparison between the x-ray emission from two symcaps with similar laser and hohlraum conditions showing a shape that reproduces within the $8 \%$ accuracy required for symcap implosions. At peak emission time, shot $\mathrm{N} 110214$ had $\mathrm{P}_{0}=65 \pm 8 \mu \mathrm{m}$, $\mathrm{P}_{2} / \mathrm{P}_{0}=12 \pm 7 \%$ and $\mathrm{N} 110612$ had $\mathrm{P}_{0}=60 \pm 15 \mu \mathrm{m}, \mathrm{P}_{2} / \mathrm{P}_{0}=2 \pm 8 \%$. The images show effective 

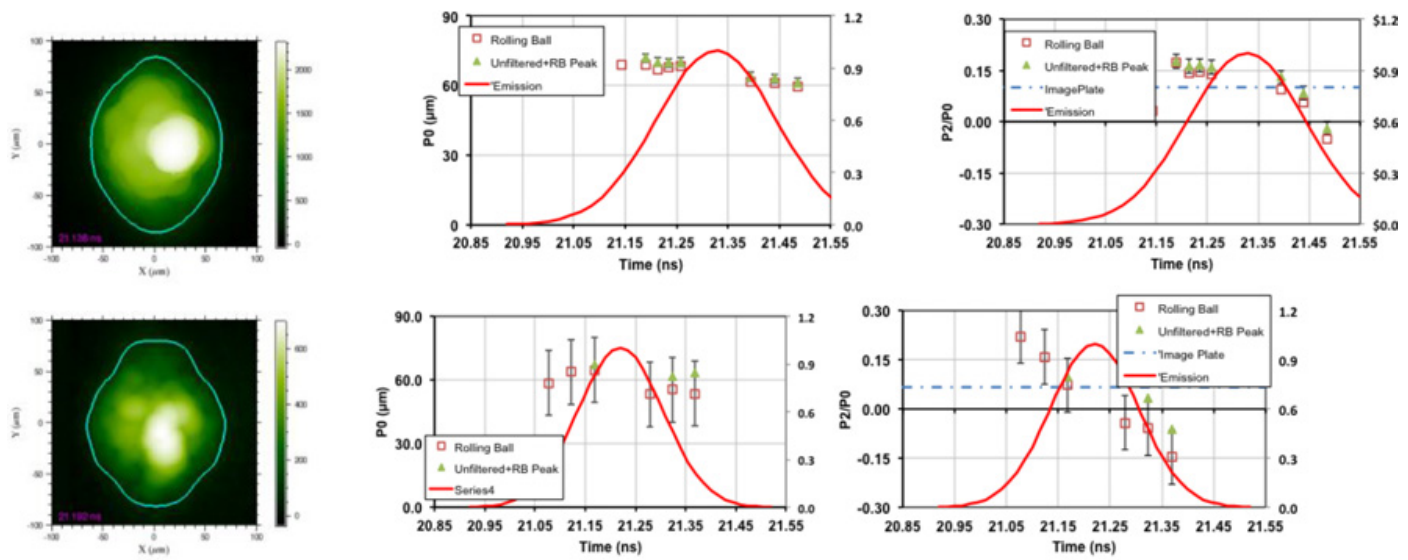

Figure 1. Symcap experiments N110214 [top line] and N110612 [bottom line] showing the equatorial X-ray images near peak emission [left frames], the time history of the radius of the x-ray emission [middle frames], and time history of the second order Legendre Polynomial Amplitude [right frames]. The triangles are for measurements at the $17 \%$ contour estimated using no filtering of the image, while the squares are for the estimate using a rolling ball technique to reduce the effect of any hot spot due to emission from Ge that is mixed into the image. The red line shows the fitted shape of the total $\mathrm{x}$-ray emission inside that contour.
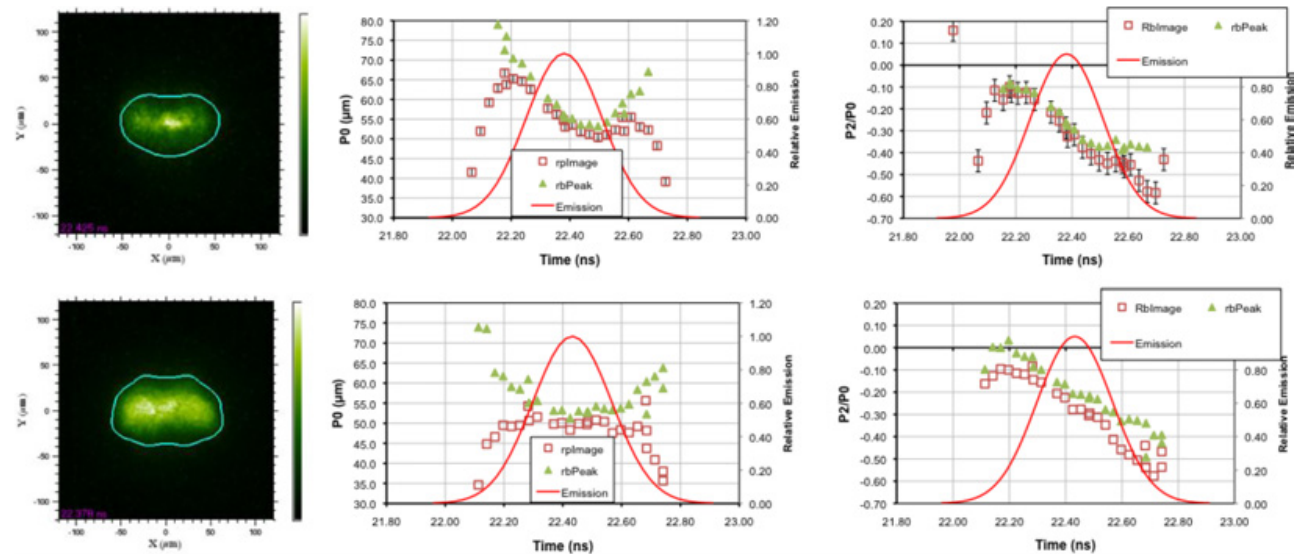

Figure 2. Symcap N110807 with Ge doping [top line] with the corresponding symcap shot N110821 with Si doping [bottom line] showing the equatorial x-ray images near peak emission [left frames], the time history of the radius of the $\mathrm{x}$-ray emission [middle frames], and time history of the $\mathrm{P}_{2} / \mathrm{P}_{0}$ [right frames]. The triangles are for the $17 \%$ contour estimated using no filtering of the image, while the squares are for the estimate using a rolling ball technique. The red line is the fitted shape of the x-ray emission inside that contour.

removal of the Ge emission. This will be compared in the next section to Si doped implosions, where no Ge was present.

Figure 1 shows the time dependent variation of the radius for these two shots using this analysis. Both show the emission radius decreasing with time, with the $\mathrm{x}$-ray emission peak occurring before the minimum in radius, indicative of the effect of the small cone fraction variation among the shots as well as. The figure shows two such measurements of the radii, with a roller ball and with a filtered image. The data without the error bars is analyzed using a roller-ball filtered image. The data with error bars uses the original image with the peak determined from the roller ball filtered image. The difference between the two analyses is smaller than the quoted error. The measurement of the normalized second order 
Table 1. Comparison of the Ge and Si doped shapes around x-ray bang time.

\begin{tabular}{|l|c|c|c|c|}
\hline & N110807 Ge Doped & Uncertainty & N110821 Si Doped & Uncertainty \\
\hline $\mathrm{P}_{0}$ & $49 \mu \mathrm{m}$ & $+/-2.4 \mu \mathrm{m}$ & $53.42 \mu \mathrm{m}$ & $+/-3 \mu \mathrm{m}$ \\
\hline $\mathrm{P} 2 / \mathrm{P} 0$ & $-25.9 \%$ & $+/-6 \%$ & $-31.2 \%$ & $+/-5.4 \%$ \\
\hline $\mathrm{P} 2$ & $12.7 \mu \mathrm{m}$ & $+/-3.5 \mu \mathrm{m}$ & $17 \mu \mathrm{m}$ & $+/-4 \mu \mathrm{m}$ \\
\hline $\mathrm{P} 4 / \mathrm{P} 0$ & $1.1 \%$ & $+/-1 \%$ & $3.2 \%$ & $+/-2.7 \%$ \\
\hline $\mathrm{P} 4$ & $0.5 \mu \mathrm{m}$ & $+/-0.5 \mu \mathrm{m}$ & $1.7 \mu \mathrm{m}$ & $+/-1.5 \mu \mathrm{m}$ \\
\hline
\end{tabular}

polynomial $\mathrm{P}_{2} / \mathrm{P}_{0}$, shown in figure 1 , shows a greater variation with time. However the uncertainty in measuring the correct time axis for the N110612 was larger than for N110214. Time-integrated images, using an image plate surrounding the gated instrument, also showed that symmetry is reproducible within the specification of a symcap $\mathrm{P}_{2}$ symmetry of $+/-8 \%$.

\subsection{Comparison of Ge and Si doped symcap capsule implosions}

Ge had been doped into the capsule ablator to reduce the effect of preheat on the inner $\mathrm{CH}$ layer of an ignition capsule to keep a low Atwood number. However experiments that measure the implosion velocity found that using a Si doped capsule may give a slightly faster implosion velocity. We took advantage of the use of Si shot N110821 to evaluate the difference with a similar symcap shot N110807 with $\mathrm{Ge}$, thus evaluate the technique to remove spots due to Ge. Si-dopants do not emit lines in the detector bandwidth.

The capsules were shot in a 5.75-mm diameter, 10.01-mm long gold hohlraum with a $57 \%$ laser entrance window and wavelength separation of $4.5 \AA$ between the inner and outer cones. The hohlraums were filled with $0.96+/-0.0045 \mathrm{mg} / \mathrm{cc}$ of He to hold the gold wall from ablating into the capsule. The Ge/Si doped capsule shots had laser energy of 1.31 and $1.326 \mathrm{MJ}$, external foot CF of 0.18 and 0.1 , and the same peak CF of 0.35 and 0.344 , respectively. Table 1 shows that the Ge-symcap implodes to a radius of $49+/-2.4 \mu \mathrm{m}$, while the Si-symcap implodes to a close radius of $53+/-3 \mu \mathrm{m}$. However, the change in $\mathrm{P}_{2} / \mathrm{P}_{0}$ remains within uncertainty, and the $\mathrm{P}_{4} / \mathrm{P}_{0}$ is small and shows a smaller difference. The X-ray images are similar, confirming that a Si doped shell implodes without the bright spots seen in a Ge doped capsule, and the effective removal of the bright spots from the Ge doped capsule images. The different swing in time was due to the difference in foot CF.

\section{SUMMARY}

We have shown that using symcaps at widely separated time with similar hohlraum and laser conditions gave, within the accuracy of the measurement, similar x-ray shapes. Also, the core emission shapes from symcaps with $\mathrm{Si}$ and Ge doped capsules gave similar shapes. Results from these experiments have been used to create shape-tuning curves [13], and to calculate core size.

\section{References}

[1] M. C. Herrmann, M. Tabak, and J. D. Lindl, Phys. of Plasmas 8, 2296 (2001)

[2] N. Delamater et al., Phys. Plasmas 7, 1609 (2000)

[3] G. A. Kyrala, J. L. Kline, S. Dixit et al., Phys. of Plasmas 18, 14 (2011)

[4] O. L. Landen, J. Edwards, S. W. Haan et al., Phys. Plasmas 18, 051002 (2011)

[5] N. Izumi, C. Hagmann, G. Stone, G, et al., Rev. Sci. Instruments 81, 10E515 (2010)

[6] S. H. Glenzer, B. J. MacGowan, P. Michel et al., Science 327, 1228 (2010)

[7] P. Michel, S. H. Glenzer, L. Divol et al., Phys. of Plasmas 17, 6 (2011) 


\section{IFSA 2011}

[8] G. A. Kyrala, S. Dixit, S. Glenzer et al., Review of Scientific Instruments 81, 8 (2010)

[9] S. W. Haan, J. D. Lindl, D. A. Callahan et al., Phys. Plasmas 18, 051001 (2011)

[10] C. A. Haynam, P. J. Wegner, J. M. Auerbach et al., Appl. Opt. 46, 3276 (2007)

[11] E. Moses and C. R. Wuest, Fusion Sci. Tech. 47, 314 (2005)

[12] B. A. Hammel et al, Phys. Plasmas 18, 05630 (2011)

[13] J. L. Kline et al, these proceedings 